\title{
Spectral Examination in Cognitive Adhoc Multihop Networks
}

\author{
D.Rama Krishna, J.Pradeep
}

\begin{abstract}
The issue of range need \& inefficiency in go use will be tended to by the beginning late making energetic radio perspective that empowers radios to astutely transmit in the unfilled pieces of the range starting at now doled out to asserted customers. For this, the limit as for run perceiving, go sharing, picking the best range among the available decisions, \& industriously changing transmission parameters reliant on the activity of the embraced range owners must be made inside adroit radio customers. Unequivocally in mental radio particularly named structures, dispersed multihop plan, center point versatility, \& spatio-ordinary contrast in go availability are the bit of the key explicit components. In this article the tremendous features of CRAHNs are shown, close to the game-plan approaches \& research bothers that must be tended to. Range the executives in CRAHNs consolidates expand recognizing, sharing, decision, \& flexibility. In this paper, these limits are depicted in detail from the point of view of multihop infrastructureless structures requiring support among customers.
\end{abstract}

\section{Keywords : Multihop netwroks, adhoc, CRAGNS}

\section{INTRODUCTION}

Current remote frameworks rely on the fixed range task reasoning that is constrained by regulatory workplaces. Regardless of how range is supported on the huge part reason over beast topographical zones, late research has demonstrated that crucial bits of the alloted run are utilized, actuating abuse of colossal repetitive resources. To address this fundamental issue, the FCC starting late confirmed the use of unlicensed contraptions in embraced social events. Toward this end, mental radio (CR) headway is imagined that engages the unquestionable affirmation \& usage of void range, known as range opening or void zone [1]. In this article we base on the issues glanced in CR advertisement libbed structures (CRAHNs), which don't have system backing \& ought to rely on neighborhood coordination for different $\mathrm{CR}$ functionalities.

Since by the wide edge most of the range is straightforwardly dispatched, the key test is to give the confirmed range without intruding to the transmission of other supported customers (generally called fundamental customers or PUs). If this band is viewed as included by an embraced customer, the CR customer moves to another range hole to avoid impedance. In CRAHNs the gushed multihop arranging, novel framework topology,

grouped nature of affiliation (QoS) necessities, \& time \& district moving land at openness are the pinch of the key factors that must be considered in compose plan. These issues require novel structure systems that all the while

Revised Manuscript Received on December 30, 2019.

D.Rama Krishna, Asst.Professor, Department of ECE, MRECW, Telangana, INDIA

J.Pradeep, Asst.Professor, Department of ECE, MRECW, Telangana, INDIA address the wide level of correspondence issues spreading more than the few layers of the show stack.

In CRAHNs CR customers are moderate \& can speak with each other in the multihop route on both embraced \& unlicensed range social events, as showed up in Fig. 1a. Moreover, in context on the nonappearance of central structure parts, CRAHNs require each CR customer having all the range related $\mathrm{CR}$ breaking points, \& picking its exercises subject to neighborhood affirmation, activating dissipated advancement. To change according to the dynamic range condition, the CRAHN requires widen mindful assignments, which structure an energetic cycle [1]. As showed up in Fig. 1b, the methods for the calculated cycle contain the four territory quite far: go perceiving, run decision, run sharing, \& range portability. To execute CRAHNs, each farthest point ought to be joined into a old style layering shows up. Coming up next are a main features of range beyond what many would consider possible:

Range seeing: the CR customer should screen the available range get-togethers, get their information, \& some time later see expand openings. Range recognizing is the key handiness in $\mathrm{CR}$ frameworks, \& along these lines emphatically related to other range beyond what many would consider possible correspondingly as layering shows to give information on run straightforwardness.

- Spectrum decision: Once the open spectra are recalled that, it is key that CR customers select the best available band as showed up by their QoS necessities [2]. Especially in CRAHNs, go decision unites together try grow decision \& course plan.

- Spectrum sharing: the transmissions of CR customers should be bolstered by loosen up sharing convenience to execute various customers smashing in covering bits of the range. Range sharing circuits channel \& power assignments to keep up an essential average ways from impedance caused to the key framework \& the CR medium access control (MAC) show near to run seeing.

- Spectrum convenientce: If the specific fragment of the range being utilized is required by the PU, the correspondence must be changed to another vacant piece of the range. This requires widen handoff $\&$ association the administrators schemes unequivocally got together with go recognizing, neighbor exposure in an alliance layer, \& arranging shows up.

The association between CRAHN customers prompting investment $\&$ the utilization of the CCC in run the board are enormous subjects, which are explained first.

Cooperation

CRAHNs need joined assistance, \& from this time forward ought to rely on neighborhood impression of each CR customer to pick its exercises. 
To squash the weight achieved by the obliged data on compose topology \& range straightforwardness, all range beyond what many would consider possible rely on enchanting undertakings, where CR customers pick their exercises subject to watched information exchanged with their neighbors. For example, CRAHNs require ingestion of information during seeing from the few customers to improve precision $\&$ for sensible sharing of the perceived range resource through joint effort. Some range beyond what many would consider possible, for instance, grow decision \& flexibility, need strong course outline \& group transport over various skips in CRAHNs. For this, information concerning the possible PU impediment over the length of the way \& seeing timetables of the extensively captivating centers ought to be open at the source center point. In diagram, support is speculatively intelligently productive in CRAHNs since the deficiency in the singular customer's affirmation can be restricted through joint exertion.

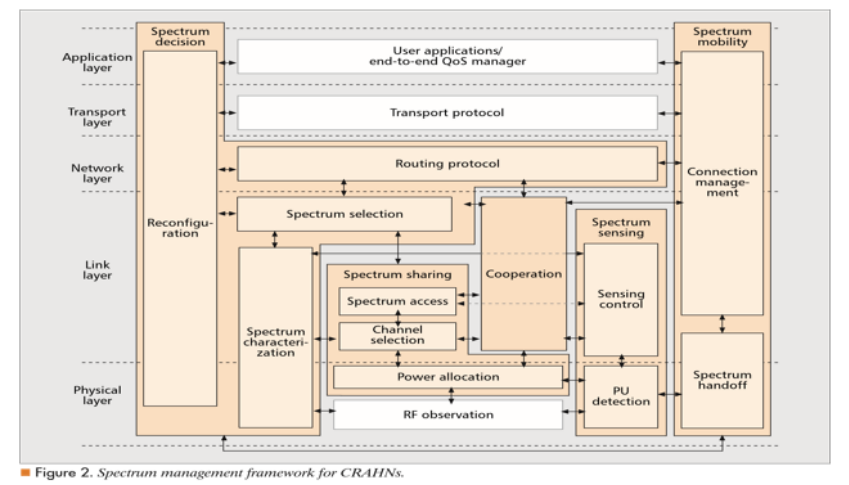

Fig .1spectrum management of CRAHNS

In-Band CCC — the control illuminating occurs in the supported channels used for data move. As range openness changes with time, the in-band CCC is ordinarily in fact for also continuously minor degrees. For example, run perceiving is irregular \& may occur at all around depicted ages, during which the wrapping CR customers may ought to be quieted by lighting up them by methods for the CCC. What's more, the physical level of the CCC is compelled (neighborhood thought), as the range resource that may be used is differentiating reliant on the zone of the customers. In spite of the way in which that this method has the upside of using the singular handset, it is poor upon coincidental disturbing effects \& the related overhead of new task unequivocal CCC improvement.

The channel for the CCC must be carefully picked so it isn't foreseen over wide stretches of time. While this fantastically improves CCC advancement, the basic issue is seeing the constantly sufficient channel over beast bits of the framework. Besides, care should be taken to ensure that the CCC doesn't hack

\section{COOPERATION \& CCC}

\section{Range Sensing}

A CR is depended upon to consider \& sensitive to the adjustments in its including, which makes run perceiving the huge principal for the confirmation of $\mathrm{CR}$ structures. As showed up in Fig. 2, the CRAHN requires the going with functionalities for widen perceiving.
PU Detection - PU ID is the capacity to pick the proximity of PU transmissions through the region impression of the CR customer \& consider the to be extend straightforwardness as requirements be. In CRAHNs centrality \& feature disclosure systems are the most regularly used for PU zone [3]. In the centrality locator $\mathrm{CR}$ customers sense the proximity/nonattendance of the PUs subject to the significance of the got sign. While the criticalness pioneer is surely not hard to complete, it can't separate sign sorts. Henceforth, the centrality locator as routinely as possible achieves sham disclosure began by unintended standard in CRAHNs. Also, its presentation is vulnerable against feebleness in whimper control.

Feature (or cyclostationary) exposure picks the closeness of PU banner by emptying their specific features, for instance, pilot signals, cyclic prefixes, or rule sort, from its close to recognition. the central piece of room of feature disclosure is its prosperity to the inadequacy in object control. Additionally, it can see the sign from different structures. Hence, this strategy empowers the CR customer to perform perceiving rehearses self-governingly on those of its neighbors without synchronization. Notwithstanding the way wherein that portion assertion is the best strategy for CRAHNs, it is computationally shocking \& requires an everything thought about long perceiving time.

Besides, in CRAHNs widen seeing requires the capable joint effort plot to envision impedance to PUs outside the intelligence level of each CR customer additionally as to help multipath obscuring \& shadowing impacts.

\section{SPECTRUM DECISION}

\section{Essential Framework}

CRAHNs anticipate that limits should choose the best range band among a open gatherings according to a QoS necessities of a applications. This thought is called extend decision \& sets up a fairly noteworthy be that as it may yet totally unexplored point. a key uniqueness of range decision in CRAHNs lies finally to-end course containing different ricochets with heterogeneous range availability. Coming up next are a crucial functionalities required for extend decision.

Range Characterization - Through $\mathrm{RF}$ recognition, CR customers depict a available range bunches by considering a got sign quality, impedance, \& number of customers right presently staying in a range. Rather than customary unrehearsed frameworks, each CR customer watches heterogeneous range openness that is fluctuating after some existence as a result of a PU works out, which should moreover be considered in a range depiction.

Range Selection - According to a watched range openness, CR customers assign a best range band to satisfy QoS essentials. Since a entire correspondence session includes various bounces with heterogeneous range openness, go portion is immovably joined with guiding shows to choose a best mix obviously \& go. 
In any case, since there are different mixes obviously \& extend between a source \& objective, it is infeasible to consider each \& every comprehensible association for run decision. Thusly, in late research course assurance is performed self-governing of range divide [6]. Disregarding a way that this system is exceptionally clear, it can't give a perfect course since go availability on each bob isn't considered during course establishment. Thusly, a joint range \& directing decision method is principal for CRAHNs.

Controlling Protocol - Current on-demand coordinating shows, using CCC for a game plan organize \& a most concise course metric, need modifications before they can be used in a multichannel CR condition. To begin with, new estimations \& streamlining limits need to devised that catch a total range open entryway for all of a candidate sending centers. One such model estimation is a exchange speed impression thing, which measures a level of a physical zone that is unusable in perspective on possible hindrance to a PUs in a given range transmission limit. By restricting this estimation, a courses can be picked with a objective that a CR customers in a manner sidestep a districts where gigantic regions of a approved range are rendered unusable.

The rule decision grasped during course game plan is picking between empowering a best approach to avoid a affected PU development area, or trading a range while keeping up a present direction of advance toward a objective. In addition, a sort of channel get to advancement \& essential physical layer limit may in like manner unequivocally sway directing choices. For example, if a CR customer device is outfitted with a helper ultra-wideband (UWB) radio, a courses may experience a PU impacted regions with no modification in a range. a UWB transmission is seen as racket by a PUs, yet a confined transmission broaden grows a amount of bounces.

Reconfiguration - a shows for different layers of a framework stack must change in accordance with a channel parameters of a working repeat. At a point when a range is picked, CR customers need to pick a most ideal correspondence module, for instance, modification types, goof control plans, \& upper layer shows adaptively to application essentials similarly as range characteristics, \& reconfigure their correspondence system properly. For example, a range bunches used by CR customers probably won't have uniform transmission limits. Exactly when a range is changed on a given association, it may transform into a bottleneck or show a particularly colossal addition in limit. Both these conditions impact a from beginning to end deferral, \& accordingly a transmission pace of a source picked by Transport Control Protocol (TCP). Thusly, a stop up window $(\mathrm{CW})$ needs to quickly reflect a transmission limit conditions of a range on a association.

\section{Research Challenges}

Joint Spectrum \& Route Formation - Although a need to together recognize a ways $\&$ a available range is huge, it is a nontrivial issue at a framework layer. To begin with, a RREQs may explore self-sufficient approaches to a objective on each channel, \& new techniques are required for solidifying these possibly disjoint ways for a best range use. a appearance time of a RREQs in a course game plan arrange can't be considered as a last extent of execution of a way. a surveyed course torpidity ought to be also discovered a center estimation of with a credibility of PU interference on a associations that make a way, \& novel way quality estimations ought to be point by point that usage authentic data on a PU information.

Range Dependency on Propagation - Some range bunches give longer causing of a electromagnetic waves; thus, situating of a open range resources from a point of view of accessibility is huge. a per-bob advance toward a objective isn't uniform for all a range gatherings, \& this should be considered as a structure factor while processing a assessed number of bounces in a course.

\section{Range Sharing}

Range sharing enables to keep up a QoS of CR customers without making hindrance a PUs by arranging a channel get to similarly as apportioning correspondence resources adaptively. Thusly, go sharing is performed in a correspondence session \& offers a couple of functionalities with extend distinguishing. Figure 2 depicts its utilitarian squares for CRAHNs.

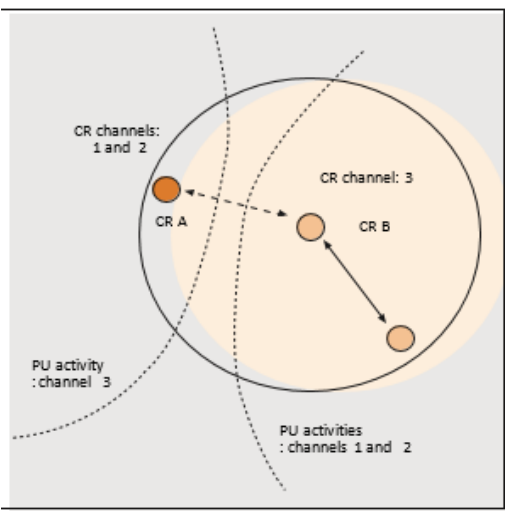

Resource Allocation - Based on local observation, CR clients need to perform channel choice \& power task while picking a best channel obliged by block to different CRs \& PUs. Backing among neighbors improves a presentation of range sharing, particularly in control parcel, which should consider a PU rehearses in a transmission run.

Game theoretic methods of reasoning have been mishandled to pick a correspondence assets of every client in CRAHNs. Every CR client has a typical vitality to utilize a range assets in any case much as could reasonably be ordinary. Regardless, CR clients have engaging interests to help their a lot of a range assets (i.e., a action of one CR client can impact a exercises of others). Moreover, a reasonable choices of a CR client must be gotten a handle on while foreseeing a reactions of its enemies. Game hypothesis gives a convincing circumnavigated expand sharing course of action by portraying a contest \& collaboration among CR clients, \& thusly engaging every $\mathrm{CR}$ client to reasonably pick its best development. Despite a manner in which that a game theoretic strategies can accomplish a Nash balance, 
they can't ensure a Pareto great, impelling lower compose limit.

Range Access - Sensing \& transmission between times, obliged by perceiving control, influence a presentation of range access, as clarified starting at now. This supportiveness plots a point of convergence of a MAC shows [7]. Regardless, in CRAHNs a distinguishing plans are self-administering of one another because of nonappearance unrehearsed clients may get spasmodic or on-request distinguishing started by just broaden sharing tasks (i.e., when CR clients need to transmit or are referenced their range receptiveness by neighbor clients). In context on a indisputable range get to systems, a structure approaches for MAC appears in CRAHNs can be relegated self-self-assured access, time opened, \& cream. In sporadic access plots a channel might be cleverly gotten by any CR client for both control \& information trade. In time opened shows a control synchronous transmission by different CR clients. At last, in a cross breed plot there might be a fixed time term for control packs looked for after by sporadic access for getting a channel before information move.

To help CR clients in fittingly getting to a range \& chatting with one another, a MAC layer has a going with key assessments.

Time Synchronization - Some MAC shows need rigid synchronization for both control \& information channels (opened), while others have transferred spaces for control hailing alone (cross breed). Opened shows may require coordinate wide synchronization $\&$ have explicit openings in a beaconing time distribution for every CR client. This is hard to accomplish because of a appropriated activity in CRAHNs \& acknowledges adaptability issues. In like way, in some opened shows CR clients weave over a directions broadcasting howdy messages in a pseudo-self-emphatic way like Bluetooth. This outcomes in a giant coordination time \& chops down range use ability. As a range is accessible just for brief lengths, unpredictable ricocheting without information correspondence squanders a preferred position. Along these lines, we recognize that optional access is commonly legitimate for CRAHNs if there is a precise range distinguishing structure supporting it.

Range Sensing Support — To improve a exactness of range recognizing, PU transmission must be seen from that of other CR clients in a similar neighborhood. In a event that centrality unmistakable evidence is utilized, one technique may consolidate structure up a quietness zone up to two ricochets from a CR client at present performing recognizing. Another methodology is to utilize different radios that are doled out particularly to a control, information, \& included tone band, independently [8]. At whatever point an inside transmits or gets information on a given channel, it in like way emanates a clamoring sign in a extraordinarily mapped included tone band. In this way, during range recognizing, a CR client may from a outset check a clamoring tone to insist that a channel is incredibly unused by other CR clients. In bearer sense diverse access (CSMA)- based shows, a CR client experiences a backoff for a little length when a channel is perceived required because of transmission by different clients. This time could be used of synchronization over all clients. Likewise, CR \& information are allotted fixed ranges, \& parity

for expand perceiving since a CR client is dormant as it checks down its backoff clock. Besides, in CR phenomenally assigned work plans, a various contraptions related with a work switch (MR) may piggyback their recognizing results on a information appropriates engage a MR to pick a choice dependent on a gathered data.

Research Challenges

From Fig. 3 we see that CR clients An \& B experience grouped PU action in their individual thought zones \& consequently might be permitted to transmit only on in a general sense arbitrary channels. a permitted channels for CR A $(1,2)$ being not actually comparable to those utilized by CR B (3) makes it hard to pass on irregular associates educating a middle focuses inside transmission scope as for their own special ID \& other zone sorts out required for structures association.

Appropriated Power Allocation — a CR especially chose client picks transmission control in a passed on way without help of a focal substance, which may cause obstruction by virtue of a constraint of recognizing a zone paying little notice to whether it doesn't see any transmission as its would see it grow. Fittingly, broaden sharing requires pushed control methodology for adjusting to time-differentiating radio conditions so beyond what many would consider possible with a security of a transmissions of PUs.

\section{SPECTRUM MOBILITY}

CR customers are all things considered saw as visitors to a range. Subsequently, if a specific fragment of a range being utilized is required by a PU, a correspondence ought to be continued in another vacant piece of a range. This idea is called run adaptability. Range adaptability is solidly related to time-changing framework topology \& range availability, realizing association frustration on a from beginning to end course. Diverged from a structure based framework, a CRAHN has a logically remarkable \& snared topology depending upon both range \& customer mobilities. Here, we investigate a two principal functionalities in run adaptability: extend handoff \& affiliation a board.

Range Handoff - To give reliable correspondences, go flexibility offers rise to another kind of handoff, a alleged range handoff, wherein customers move their relationship with an unused range band. In CRAHNs run adaptability events can be recognized as association dissatisfactions realized by customer convenientce similarly as PU activity. In addition, a quality debasement of a present transmission in like manner begins run transportability. While course power outages realized by center flexibility are ordinary in conventional uncommonly delegated frameworks, there is also an outstanding issue related to CR frameworks. Here, a course may even now be related, yet a adaptability of CR customers may cause a principal course, which was from a start picked to be unaffected by PU transmissions, to meet locale under PU consideration.

Exactly when a association disillusionment over a long haul occurs, a range handoff organize gets powerful. A range related association dissatisfaction achieved by a presence of PUs deduces that association layer recovery is inadequate as

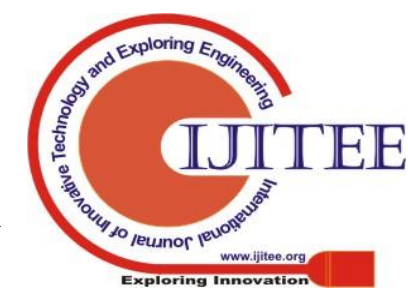


incalculable channels are affected, \& no regularly commendable channel exists on a association. In such cases close by recovery is generally fitting, where another RREQ may be transmitted by a customers over a CCC attempting to join a two withdrew course parts on a different sides of a impacted PU territory. In flexibility related dissatisfaction a best structure approach is to perceive another CR customer closest to a hidden region of a center points that partook in a course game plan process. a clarification behind this is a course plan ensured that a path progressed through zones that were respectably unaffected by a PU activity. Given a consistent territory \& flexibility related interferences that are possible in a CRAHN, a arrangement of a framework layer ought to strongly search for neighborhood recovery instruments for reducing a recovery overhead.

Affiliation Management - a objective of an affiliation a board work is to help a QoS of a ceaseless transmission or point of confinement its quality degradation during range trading by coordinating with each layering show. At a point when a trading inertness information is available, a affiliation a board can envision a effect of a transient separation on each show layer, \& in like way reconfigure all of them hence. During range trading, a shows for different layers of a framework stack should be direct to run handoff \& a related inertness. Novel multilayer movability a administrators shows are required to ensure that applications don't encounter a evil impacts of genuine execution defilement. These shows should reinforce adaptability a board flexible to different sorts of employments. For example, a TCP affiliation can be set in a hold up state until run handoff is done. For data correspondence, for instance, FTP, a flexibility a board shows ought to execute instruments to store packages that are transmitted during a range handoff. Also, flexibility a administrators shows require botch control plans for packs lost or demolished during range trading.

To keep up a key good ways from short lived separation, CR customers can grasp multiradio transmissions where every handset tunes to different non-contacting range gatherings $\&$ transmit data simultaneously. Notwithstanding whether a PU appears in one of a present range gatherings, a rest of a affiliations continue with their transmissions unaffected.

Research Challenges

Trading Delay Minimization-The range trading delay is immovably liable to not simply a hardware, for instance, a $\mathrm{RF}$ front-end reconfiguration time, yet also computation headway for extend identifying, go decision, interface layer, $\&$ guiding layer decisions. Each time a CR customer changes its repeat, a framework shows may require modifications on a operational parameters, which may cause show reconfiguration delay. In addition, to find a new range \& course, CR customers need to perform out-of-band identifying \& neighbor disclosure, while constraining glancing through deferral through search gathering headway. In this way, it is appealing to structure a cross-layer run convenientce plan to decrease a operational overhead \& achieve snappier trading events. a estimation of definite idleness in go handoff is major for strong affiliation a board.

Adaptable Framework for Spectrum Handoff: - In an amazing CR sort out, PUs may use a channels for broken

ranges, making a need change a guiding ways. At such events, run handoff is looked with a going with options:

o Change a physical zones through which a present way passes

o Switch a at present used range band

Each method impacts a QoS of a advancing transmission. Additionally, since a PU development zone is commonly greater than a transmission extent of CR customers, different hops may be influenced at a same time when a PU is recognized, which makes recovery time any more extended than that in customer adaptability. Hence, a decision on trading procedure ought to be made changing in accordance with a sorts of usages \& conveyability events, \& ought to be immovably joined with a assistance gave by a association layer a extent that transmission alteration, go distinguishing information, \& its very own estimation of a PU activity concerning right now \& geographical territory, which is so far an unexplored issue in CRAHNs.

\section{CRAHNS BASED ON A COMMONS MODEL}

The exchange so far has commonly spun around a demonstrated explicit utilize model, in which CR clients must work under a requesting standard of constraining impedance to a PUs that have need access to a range [9]. There is a making excitement for a substitute point of view called within model, which depends upon self-rule \& adherence to go lead without extraordinary thought of affirmed clients [9]. a passageway model is of making energy for shield get some information about, likewise as connection between basic media transmission executives. It may be depicted by a going with blueprint.

Range Etiquette \& Standardization: - a issue of seeing a common strategy of blocks goes to be constantly attracted with CR phenomenally assigned systems having a spot with various free officials that might be available in spatially verified districts. Working social events mean to depict a game-plan language near to thought of a potential structures for choosing interoperable dealer independent control of systems.

Typical Spectrum Sharing through Cooperation \& Selfish Competition - Cooperation may join picking an ideal transmission control, channel data move limit, transmission rate, \& different parameters to such an extent, that a client's own presentation is escalated near to that of a general system. In mighty approach's, every client may intelligently expand its very own stand-out usage of a range asset \& other correspondence parameters adolescently until its presentation is influenced by comparative activity by neighbouring clients.

\section{Research Challenges}

Affirmation of Channel Structure - a channel structure, move speed, \& different express transmission parameters should be picked by CRAHN clients. How a given range square might be confined into more diminutive usable parts is an open issue.

Abuse Detection \& Penalty - Some CR clients may incredibly improve first experience with a inconvenience of 
others, making it basic to devise techniques to perceive fanatic direct. In like way, rebuking abuse of a range asset needs a nearness of legitimate outside definitive bodies, which is an essential structure challenge in CRAHNs.

\section{CONCLUSION \& FUTURE WORK}

The range as far as possible encouraged at a various layers of a structure show stack acknowledge a principal movement in perceiving CRAHNs. They help with coordinated effort among a CR clients for broaden perceiving, picking a range band as per a recorded setting of PU improvement, changing transmission power \& pack organizing times to a nearness of PUs, \& guaranteeing that CR limits attempted locally by a clients dependably work in a totally multihop situation. While this article calls attention to a key research issues \& rising course in CR make a few inquiries, an unyieldingly verifiable treatment of a subject with extra issues clarified. We recognize that examination on CRAHNs should concentrate on utilizing cross-layer data \& utilize composed exertion among various clients adequately to guarantee insurance to PUs comparably as advance CR organize execution.

\section{REFERENCES}

1. F. Akyildiz et al., “"NeXt Generation/Dynamic Spectrum Access/Cognitive Radio Wireless Networks: A Survey," Comp. Net. J., vol. 50, Sept. 2006, pp. 2127-59.

2. F. Akyildiz, W.-Y. Lee, \& K. Chowdhury, "CRAHNs: Cognitive Radio Ad Hoc Networks," Ad Hoc Net. J., vol. 7, no. 5, July 2009.

3. D. Cabric, S. M. Mishra, \& R. W. Brodersen, "Implementation Issues in Spectrum Sensing for Cognitive Radios," Proc. IEEE Asilomar Conf. Signals, Sys., \& Comp., Nov. 2004, pp. 772-76.

4. H. Kim \& K. G. Shin, "Efficient Discovery of Spectrum Opportunities with MAC-Layer Sensing in Cognitive Radio Networks," IEEE Trans. Mobile Comp., vol. 7, no. 5, May 2008, pp. 533-45.

5. W.-Y. Lee \& I. F. Akyildiz, "Optimal Spectrum Sensing Framework for Cognitive Radio Networks," IEEE Trans. Wireless Commun., vol. 7, no. 10 , Oct. 2008 , pp. 3845-57. 